\title{
Generation of attosecond x-ray pulses with a multicycle two-color enhanced self-amplified spontaneous emission scheme
}

\author{
Y. Ding, ${ }^{1}$ Z. Huang, ${ }^{1}$ D. Ratner, ${ }^{1}$ P. Bucksbaum, ${ }^{1}$ and H. Merdji ${ }^{2,1}$ \\ ${ }^{1}$ SLAC National Accelerator Laboratory, Menlo Park, California 94025, USA \\ ${ }^{2}$ CEA/SPAM, Centre de Saclay, 91191 Gif sur Yvette, France
}

(Received 11 February 2009; published 11 June 2009)

\begin{abstract}
Generation of attosecond $\mathrm{x}$-ray pulses is attracting much attention within the $\mathrm{x}$-ray free-electron laser (FEL) user community. Several schemes using extremely short laser pulses to manipulate the electron bunches have been proposed. In this paper, we extend the attosecond two-color enhanced self-amplified spontaneous emission scheme proposed by Zholents et al. to the long optical cycle regime using a second detuned laser and a tapered undulator. Both lasers can be about ten optical cycles long, with the second laser frequency adjustable and set to optimize the contrast between the central and side current spikes. A tapered undulator mitigates the degradation effect of the longitudinal space charge force in the undulator and suppresses the FEL gain of all side current peaks. Simulations using the Linac Coherent Light Source parameters show a single attosecond $\mathrm{x}$-ray spike of $\sim 100$ attoseconds can be produced. The second laser can also be detuned to coherently control the number of the side x-ray spikes and the length of the radiation pulse.
\end{abstract}

DOI: 10.1103/PhysRevSTAB.12.060703

PACS numbers: 41.60.Cr, 29.27.-a

\section{INTRODUCTION}

Ultrashort soft and hard x-ray sources have the potential to open new regimes in atomic and electronic processes, benefiting widespread fields in physics, chemistry, and biology. This has motivated the development of laser based ultrashort soft x-ray sources as well as the construction of fourth generation free-electron laser (FEL) sources in the soft and hard x-ray regime. Because of the duration of the electron bunch, FEL facilities have aimed for the femtosecond regime, but the formidable challenge of generating attosecond pulses is attracting much attention within the $\mathrm{x}$-ray FEL user community. Several schemes for generation of attosecond x-ray pulses have been proposed [1-9], mostly by manipulating electron bunches with extremely short laser pulses.

The two-color scheme proposed in Ref. [6] is based on the current-enhanced self-amplified spontaneous emission (ESASE) technique proposed by Zholents [10]. The electrons interact with two short laser pulses within two singleperiod wiggler magnets to generate a time-dependent energy modulation in the bunch. A dispersive section, such as a four-dipole chicane, converts the energy modulation of the electron bunch to a density modulation. The highcurrent spike formed by the overlapping peak intensities of the two lasers dominates the FEL gain process and produces an attosecond x-ray pulse. Ultrashort lasers with no more than two optical cycles are required to minimize satellite spikes. In addition, the longitudinal space charge (LSC) field in the main FEL undulator has to be considered properly [11].

In this paper, we extend the attosecond two-color ESASE scheme to the long optical cycle regime using a detuned second laser and a tapered undulator by taking advantage of the LSC field. Our scheme extends to FELs a technique proposed for high-order harmonic generation (HHG) to manipulate an electron wave packet for the generation of isolated attosecond soft x-ray pulses [12]. HHG experiments have shown a controlled detuning between the two colors can control the electron wave packet energy (low energy regime) in the subcycle domain using relatively long infrared laser pulses (up to 15 optical cycles). Here, we apply this idea to ESASE. Both lasers can be about ten optical cycles long, with the second laser frequency detuned from the first one to optimize the contrast between the central and side current spikes. A tapered undulator mitigates the degradation effect of the LSC force in the undulator [11] and suppresses the FEL gain of all side current spikes.

\section{OPTIMIZATION OF THE ENERGY MODULATION}

Our study is carried out for the Linac Coherent Light Source (LCLS) [13], but can be applied to other similar $\mathrm{x}$-ray FEL facilities as well $[14,15]$. We use similar electron beam parameters and accelerator setup as in Ref. [6], but with relatively long laser pulses of about ten optical cycles or more. A schematic setup is shown in Fig. 1. The laser system, driven by a chirped-pulse amplification

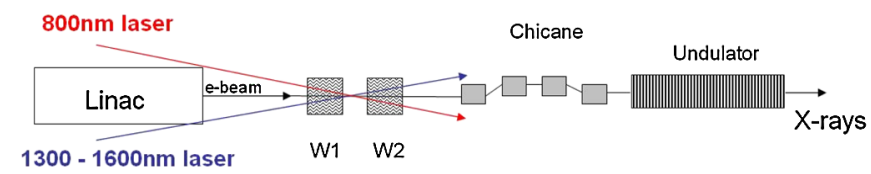

FIG. 1. (Color) Schematic setup for the two-color ESASE. 
Ti:sapphire laser, produces carrier-envelope phasestabilized pulses with a tunable central wavelength around $1.5 \mu \mathrm{m}$. The detail of this laser scheme was demonstrated in Ref. [16]. The two-color laser and two one-period wigglers produce an energy modulation on the same portion of electrons. Then a four-dipole chicane converts the electron beam energy modulation to density modulation. By optimizing the frequency of the second laser, the side peaks adjacent to the central peak of the electron bunch are expected to be small. Table I summarizes the main parameters for the electron beam, lasers, and wigglers used in this study.

The energy modulation of the electrons after interaction with a laser can be calculated from the FEL equations, as described in Ref. [6]. Here we choose the fundamental laser (laser-1) with a wavelength of $0.8 \mu \mathrm{m}$ and a pulse duration of 25 fs (intensity FWHM), which is commercially available. Laser- 1 is focused at the center of the first wiggler (W1), with its carrier wave phase adjusted to zero crossing at the peak of the laser envelope. After interaction in the first wiggler (W1) with a laser power of $20 \mathrm{GW}$, the electrons have an energy modulation amplitude of $3.8 \mathrm{MeV}$ at the center of the bunch. If we pass this beam through the chicane to optimize the density modulation for the central period, the side periods also form density peaks. We use a relatively long laser pulse here, so the side peaks in the current profile are not sufficiently suppressed by the envelope of the waveform. The current profile with laser-1 modulation after the chicane is shown in Fig. 2 with a red dotted curve.

TABLE I. Main parameters for electrons, lasers and wigglers.

\begin{tabular}{lccc}
\hline \hline & Parameter & Value & Unit \\
\hline Electron & Energy & 13.6 & $\mathrm{GeV}$ \\
& Peak current & 3.4 & $\mathrm{kA}$ \\
& rms emittance & 1.2 & $\mu \mathrm{m}$ \\
& rms energy spread & 1.1 & $\mathrm{MeV}$ \\
Laser-1 & Wavelength & 0.8 & $\mu \mathrm{m}$ \\
& $\quad$ Power & 20 & $\mathrm{GW}$ \\
& FWHM of intensity & 25 & $\mathrm{fs}$ \\
& Waist size $w_{0}$ & 178 & $\mu \mathrm{m}$ \\
Laser-2 & Wavelength & 1.314 & $\mu \mathrm{m}$ \\
& $\quad$ Power & 4 & $\mathrm{GW}$ \\
& FWHM of intensity & 45 & $\mathrm{fs}$ \\
Wiggler-1 & Waist size $w_{0}$ & 229 & $\mu \mathrm{m}$ \\
& $\quad$ Period & 0.5 & $\mathrm{~m}$ \\
Wiggler-2 & Wiggler parameter $K_{w 1}$ & 67.3 & \\
& Period & 0.5 & $\mathrm{~m}$ \\
Chicane & Wiggler parameter $K_{w 2}$ & 86.3 & \\
FEL & $R_{56}$ & 380 & $\mu \mathrm{m}$ \\
& Undulator period & 3 & $\mathrm{~cm}$ \\
& Undulator parameter $K$ & 3.5 & \\
& Average beta function & 18 & $\mathrm{~m}$ \\
& Wavelength & 1.5 & $\AA$ \\
\hline \hline
\end{tabular}

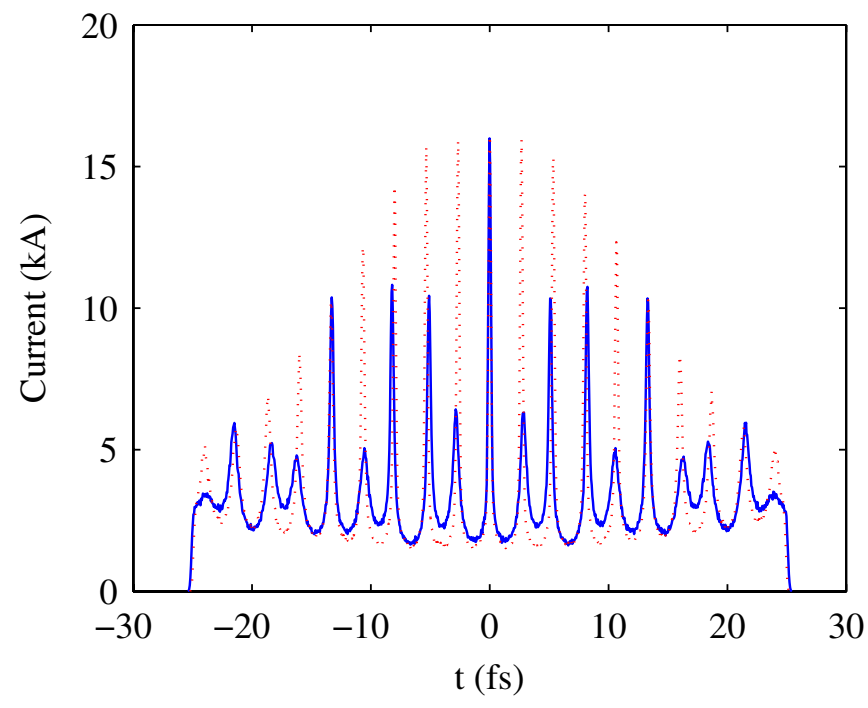

FIG. 2. (Color) Current profile after a bunching chicane. Solid blue curve is from the interaction with two optimized lasers, while the red dotted curve is from laser-1 only.

We further optimize the energy modulation by tuning the second laser (laser-2) frequency, intensity, and pulse length. The wavelength of laser-2 is optimized to be at $1.314 \mu \mathrm{m}$ with a pulse duration of 45 fs (intensity FWHM). To make an effective energy modulation, laser2 interacts at the center of the second wiggler (W2) with the same portion of the electron bunch, also at zerocrossing phase. After interacting in W2, the electrons at the bunch center gain an additional energy modulation of 1.7 MeV, while electrons at the neighboring periods lose some energy modulation due to the frequency detuning. Figure 3 shows the combined energy modulation of the electrons after interacting with the two lasers. The total

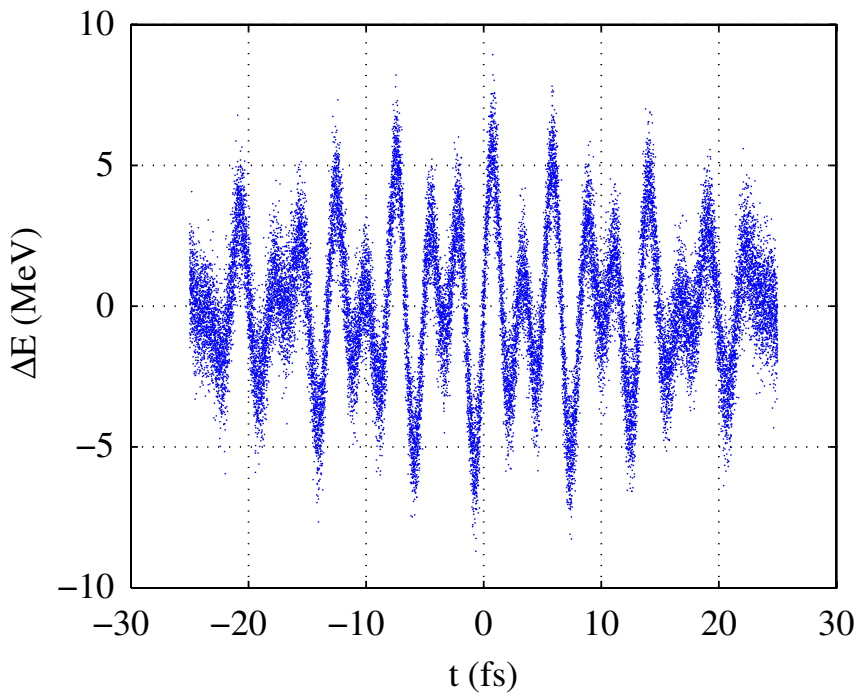

FIG. 3. (Color) Calculated longitudinal phase space of the electrons after interaction with two lasers. 
energy modulation amplitude at the center of the bunch is $5.5 \mathrm{MeV}$. The bunching chicane is set at $R_{56}=380 \mu \mathrm{m}$ to fully compress the central period of the energy-modulated bunch. The resulting current profile after the dispersive section is shown in Fig. 2 with a solid blue curve. We obtain a contrast ratio between the central peak and the side maximum peak of $17 \mathrm{kA} / 11 \mathrm{kA}$, which is much better than for the single laser case (red dotted curve in Fig. 2).

\section{LONGITUDINAL SPACE CHARGE IN THE FEL UNDULATOR}

After the bunching chicane, the energy modulation is converted to a density modulation, giving a peak current of $17 \mathrm{kA}$ in the central spike for our case, as shown in Fig. 2. Since only a small section of the bunch charge is concentrated in the spike region, the emittance growth due to coherent synchrotron radiation in the chicane and transverse space charge in the main undulator is negligible. However, due to the wiggling motion in the undulator, the longitudinal space charge field is equivalent to the free space result by changing $\gamma$ to $\bar{\gamma}_{z}$, where $\bar{\gamma}_{z}=$ $\gamma / \sqrt{1+K^{2} / 2}$, and $K$ is the undulator parameter [11]. In the limit when the electron bunch length in the average comoving frame is much larger than the transverse beam size, we can use a simplified expression to estimate the longitudinal space charge field [17]:

$$
E_{z} \approx-\frac{Z_{0} I^{\prime}(s)}{4 \pi \bar{\gamma}_{z}^{2}}\left(2 \ln \frac{\bar{\gamma}_{z} \sigma_{z}}{r_{b}}+1-\frac{r^{2}}{r_{b}^{2}}\right)
$$

where $r=\sqrt{x^{2}+y^{2}}, Z_{0}=377 \Omega, I^{\prime}(s)=d I / d s$ is the derivative of the electron current profile with respect to the longitudinal bunch coordinate $s, \sigma_{z}$ is the rms bunch length, and $r_{b}$ is the beam radius of a uniform transverse distribution. Here we take $\bar{\gamma}_{z} \sim 10000, r_{b} \approx 2 \sigma_{x} \sim$

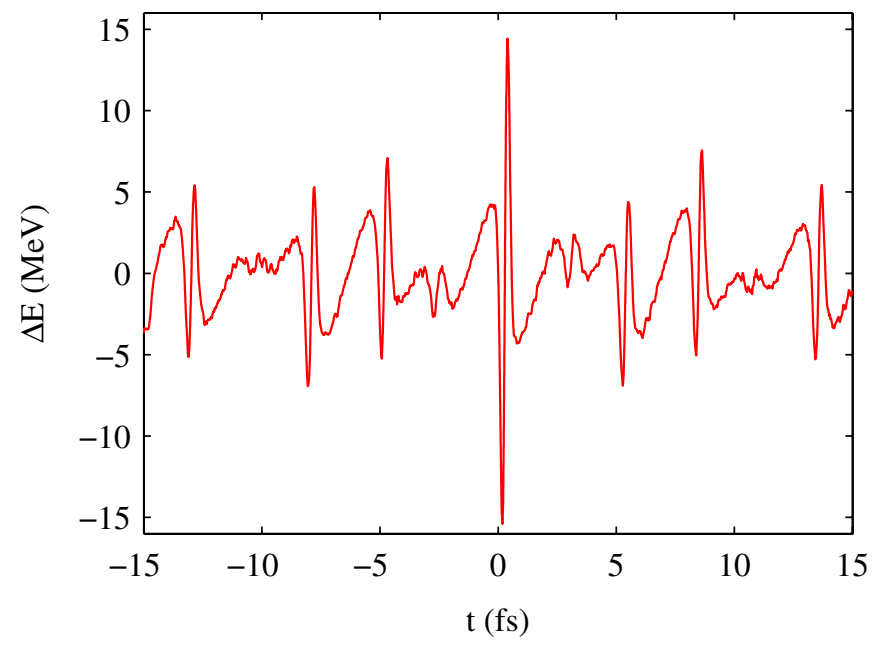

FIG. 4. (Color) Electron bunch energy modulation from the LSC field after a distance of $50 \mathrm{~m}$ in the LCLS undulator. The bunch head is to the right, as in the following figures.

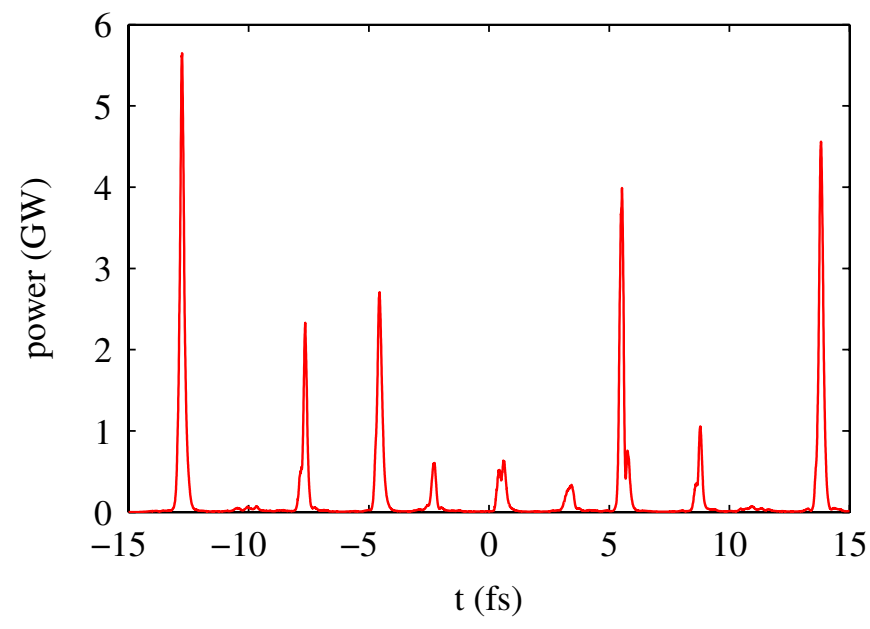

FIG. 5. (Color) A typical example of the radiation profile at an undulator distance of $50 \mathrm{~m}$ without any undulator tapering. The large energy spread induced from LSC in the bunch center degrades the FEL interaction.

$60 \mu \mathrm{m}$, and central spike $\sigma_{z} \sim 40 \mathrm{~nm}$. With these parameters, $E_{z}$ depends very weakly on the transverse position of the electrons within the beam. Hence, we drop the $r$-dependent term in computing the LSC field.

Figure 4 shows the accumulated energy modulation due to the LSC effect at a distance of $50 \mathrm{~m}$ in the FEL undulator. For the central current spike, LSC produces a strong energy chirp with a peak-to-peak energy variation of about $30 \mathrm{MeV}$, much larger than the FEL bandwidth. Such a large energy spread can degrade the FEL interaction as illustrated in GENESIS 1.3 [18] simulations that include LSC. A typical radiation profile at $50 \mathrm{~m}$ is shown in Fig. 5, where the normal undulator parameters of LCLS were used, i.e., $\lambda_{u}=3 \mathrm{~cm}, K=3.5$, and average beta function of $18 \mathrm{~m}$ in the undulator. We can see that, although the peak current at the bunch center is over $17 \mathrm{kA}$, the radiation is still suppressed due to a large energy chirp created from the LSC force.

\section{SINGLE ATTOSECOND SPIKE SELECTION USING TAPERED UNDULATORS}

It has been pointed out in Ref. [7] that a tapered undulator can compensate for the FEL gain degradation induced by a linearly chirped electron beam entering the undulator. In the case of ESASE with high-current spikes, LSC in the FEL undulator produces an almost linear energy chirp within these spikes that also grows in proportion to the undulator distance. As the radiation wave slips to the front of the current spike, the energy of the electrons in the front part of the current spike increases due to LSC and offsets the resonant condition. This is the main reason for the suppression of the FEL gain as shown in Fig. 5. By tapering the undulator parameter, $K$, we can compensate for the energy change to a large extent, and preserve the resonant 
condition for the interacting part of the electrons. However, because the strength of the LSC fields depends on the derivative of the current, the proper taper strength is stronger for the central peak than for the side peaks. In our scheme, the energy modulation for the central peak is about a factor of 2 larger than for the side ones (see Fig. 4). By choosing the taper to match the strongest chirp of the central peak, we can preserve the resonant condition in this region, while simultaneously suppressing the FEL process elsewhere in the bunch. In addition, the larger current at the central peak produces a higher FEL gain than at the side peaks. Hence, the combination of the enhanced current and undulator taper can provide a good contrast ratio between the central and side $\mathrm{x}$-ray spikes.

We use GENESIS to find the undulator taper that optimizes the contrast ratio between the central and side spikes in the radiation pulse. Tapers used to compensate for energy loss from radiation require $K$ to decrease along the undulator; in contrast, here we require an undulator taper with increasing $K$ (defined as a negative taper in [7]) to offset the energy chirp from LSC in the central current peak of the bunch. A linear taper is adopted in this study, which is defined as the relative change of the undulator $K$ over $55 \mathrm{~m}$ starting from $15 \mathrm{~m}$. The best taper to obtain a maximum power at the central spike is about $0.6 \%$; however, the contrast ratio between the central and side spikes is not good since the suppression on the side peaks is small at this taper. From simulations we obtain the best contrast ratio at $1 \%$ taper, as shown in Fig. 6, where the peak power of the first side spike is used to compare with the central one. We did five runs on each taper setup to average the peak power. At the $1 \%$ taper, the central peak power is

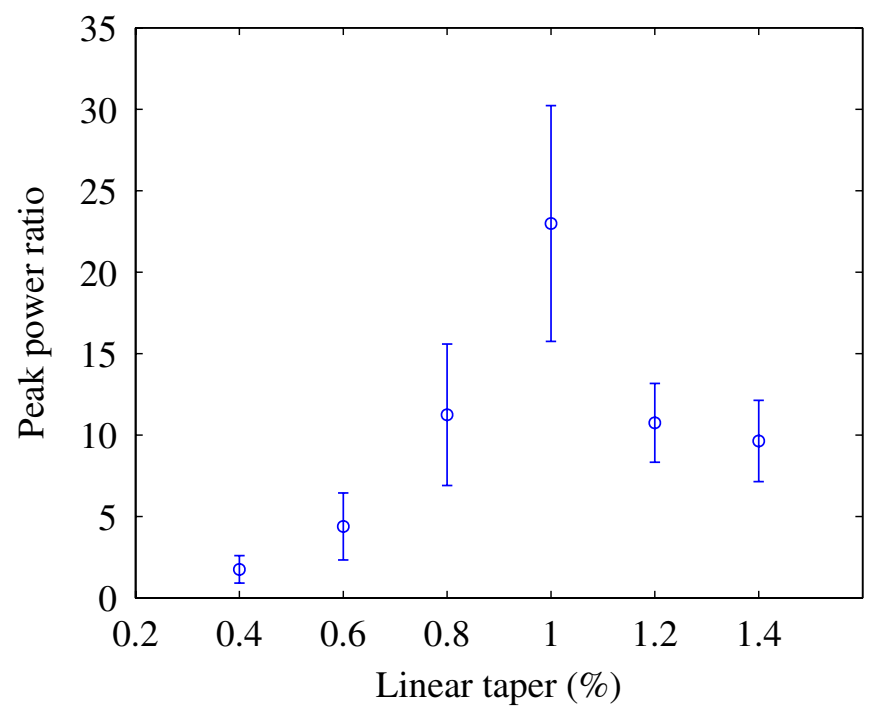

FIG. 6. (Color) The peak power ratio between the central spike and the first side one at a different undulator taper. It is a linear taper over $55 \mathrm{~m}$ starting at $15 \mathrm{~m}$. Each point is averaged over five shot noise realizations.

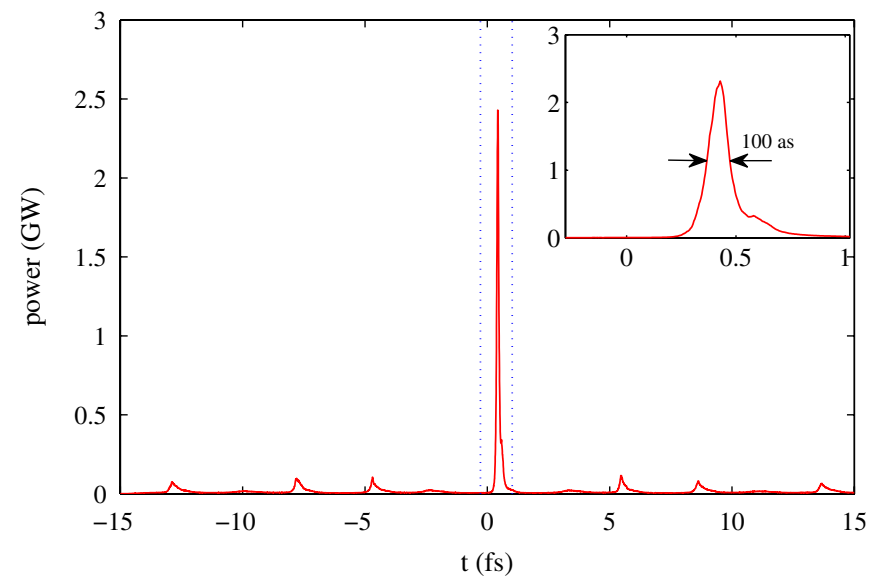

FIG. 7. (Color) A typical x-ray power profile at an undulator length of $50 \mathrm{~m}$ with an optimized undulator tapering of $1 \%$ over $55 \mathrm{~m}$ starting from $15 \mathrm{~m}$. The inset zooms in the central spike in the dotted blue region.

suppressed by about $20 \%$ while the side peak is suppressed by a factor of 7 compared with a taper of $0.6 \%$. We choose this $1 \%$ linear taper for generating a single spike in the following studies.

Figure 7 shows a typical $\mathrm{x}$-ray power profile using a negative taper of $1 \%$ over $55 \mathrm{~m}$ starting at $15 \mathrm{~m}$. We did 40 runs with different shot noise realizations on the central and one adjacent side peak to analyze the statistical fluctuations on the radiation pulse. A good contrast ratio of $\sim 20$ is obtained between the central and side spikes. The averaged peak power is $2.3 \pm 1.4 \mathrm{GW}$ at $50 \mathrm{~m}$ and the FWHM of the central spike is $102 \pm 12$ attoseconds. Since the SASE FEL starts from shot noise, the timing of the power peak also jitters with an rms of 24 attoseconds. Compared with the more uniform electron bunch of the normal LCLS configuration, the two-color ESASE scheme discussed here shows strong FEL slippage effects in the narrow current spike region. As a result, the x-ray spike duration obtained here is shorter than the typical LCLS SASE spike of 300 attoseconds, but the saturated FEL power is also reduced. For a typical LCLS bunch of $250 \mathrm{pC}$, the $\mathrm{x}$-ray pulse is about $50 \mathrm{fs}$. With the $1 \%$ taper used here at undulator length of $50 \mathrm{~m}$, the photon energy in the central spike is about half of the rest of the $\mathrm{x}$-ray pulse.

As the electron energy is strongly chirped due to LSC within the central spike, we expect the attosecond ESASE spike to be frequency chirped. This radiation chirp was discussed in $[7,19]$, where a fixed energy chirp formed before electrons entered the undulator. In our case, the electron energy chirp changes while electrons move through the undulator, complicating the radiation chirp. From GENESIS simulations, we analyzed this radiation chirp using the on-axis far field radiation. The Wigner function $W(\omega, t)$ can be constructed from the radiation field to get a time frequency "phase space" of the radiation: 


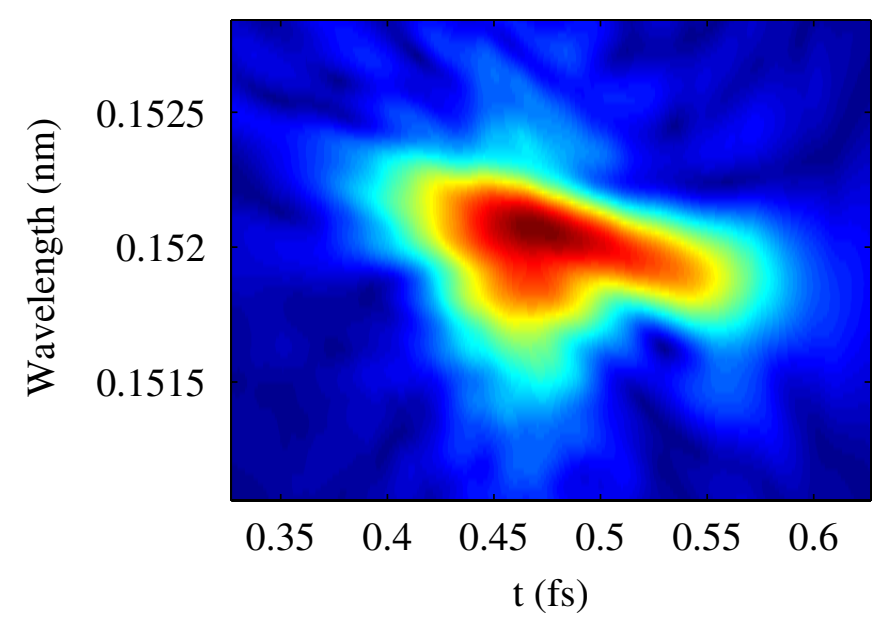

FIG. 8. (Color) False color image of the Wigner transform of the on-axis far field radiation at an undulator length of $50 \mathrm{~m}$.

$$
W(\omega, t)=\int d \tau E\left(t-\frac{\tau}{2}\right) E^{*}\left(t+\frac{\tau}{2}\right) e^{i \omega \tau} .
$$

An example calculated from Eq. (2) is shown in Fig. 8. The chirp analyzed from ten shot noise realizations is $(3.4 \pm$ $0.7) \times 10^{-2} \AA /$ fs. The frequency chirp over the attosecond pulse is larger than the intrinsic FEL bandwidth and is visible from Fig. 8.

\section{COHERENT CONTROL OF THE X-RAY RADIATION PULSE}

The second laser wavelength is optimized to be $1.314 \mu \mathrm{m}$ to obtain a single spike $\mathrm{x}$-ray pulse (single
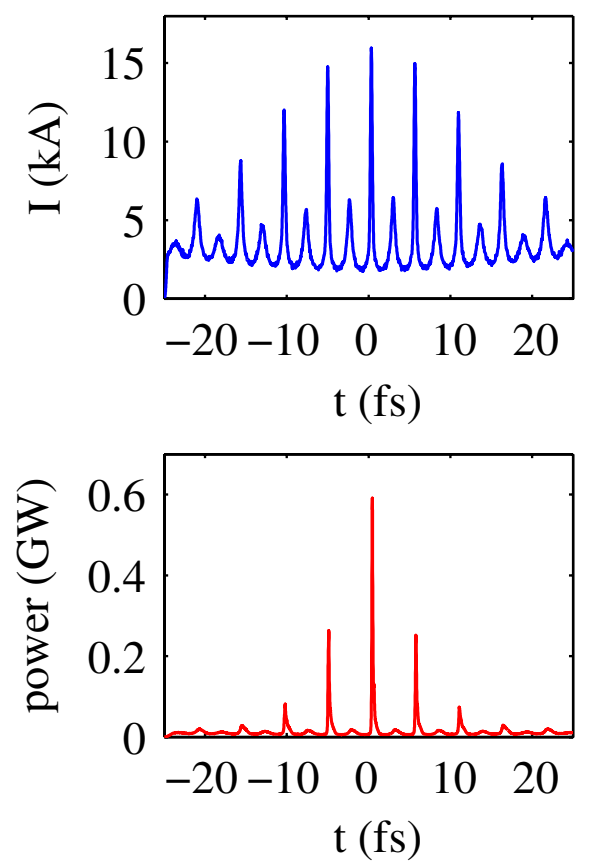

mode), as shown in Fig. 7. The laser and undulator taper are optimized to suppress the side spikes in the radiation pulse. From the laser technique, this second laser can be tunable between 1.3 to $2 \mu \mathrm{m}$ [16]. By tuning the wavelength of laser- 2 we can adjust the contrast ratio between the central and side spikes in the current profile; hence, the intensity of the side spikes in the $\mathrm{x}$-ray radiation pulse can be coherently controlled.

The taper of the main undulator is fixed at the optimized value to generate a single attosecond pulse, i.e., a negative taper of $1 \%$ over $55 \mathrm{~m}$ in our studies. Then the laser- 2 wavelength is tuned from $1.6 \mu \mathrm{m}$ down to $1.3 \mu \mathrm{m}$ to control the current profile of the electron bunch. We performed simulations at laser- 2 wavelength of 1.6 and $1.45 \mu \mathrm{m}$. Figure 9 shows the current profiles and corresponding radiation pulses at these two wavelengths of laser-2. At a wavelength of $1.6 \mu \mathrm{m}$ for laser-2, all but four of the side peaks are suppressed. Suppression of side spikes continues when tuning laser-2 down to $1.45 \mu \mathrm{m}$, then reaching the single spike mode at the wavelength of $1.314 \mu \mathrm{m}$ as described in Fig. 7 in the previous section.

\section{DISCUSSIONS AND SUMMARY}

Controlling the phase shift between the two lasers is a critical issue in the two-color ESASE scheme. Figure 10 shows an example of the radiation profiles assuming a phase shift of \pm 0.1 fs between the two lasers, with five runs for each phase shift using different shot noise realizations. Compared with Fig. 7, the central spike locations shift about $\pm 0.1 \mathrm{fs}$, and the contrast ratio between the
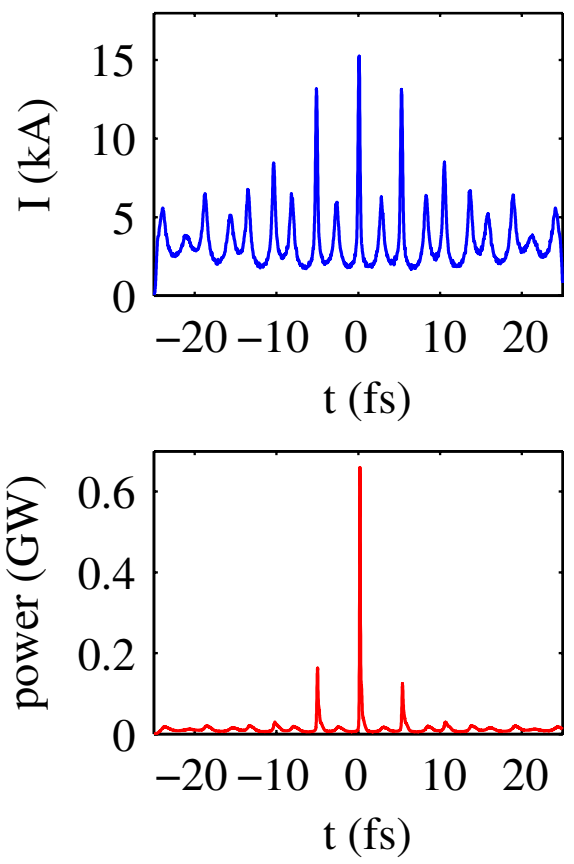

FIG. 9. (Color) Current profiles (top) and radiation pulses (bottom) versus detuning of the second laser wavelength. Left: laser-2 wavelength of $1.6 \mu \mathrm{m}$; Right: laser-2 wavelength of $1.45 \mu \mathrm{m}$. 


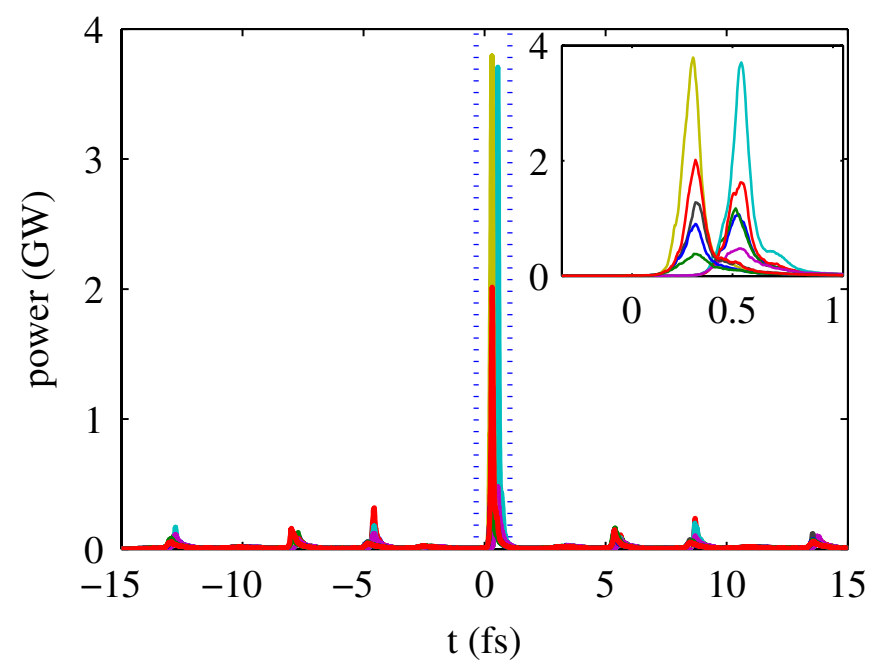

FIG. 10. (Color) Radiation profiles with a phase shift of 0.1 fs and -0.1 fs between the two lasers, five runs for each phase shift using different shot noise realizations. The inset zooms in the central spikes in the dotted blue region.

central spike and side ones is also reduced from $\sim 20$ to $\sim 10$. We note that this phase stability is intrinsically achieved in the optical parametric amplifier setup for the laser system in our considerations. The optical system is based on the generation of high-energy ultrabroadband near-IR pulses with passive carrier-envelope phase stabilization [16]. In this approach a phase stable seed is generated by difference frequency generation (DFG) between the frequency components of a supercontinuum generated by propagation in a gas-filled hollow fiber. This configuration intrinsically provides high phase stability between the different colors due to the absence of mechanical delays between the frequency components undergoing the DFG process.

In summary, we propose an attosecond x-ray FEL scheme using relatively long commercially available infrared laser pulses. This scheme builds on the two-color ESASE proposal [6] that uses extremely short laser pulses shared by other attosecond schemes. Our scheme makes use of a tunable second laser wavelength which can coherently control the number of spikes and the pulse duration of the output $\mathrm{x}$ rays. By properly taking into account the LSC field in the FEL undulator, we show that a tapered undulator can further suppress the side peaks in the radiation pulse and enable the selection of a single $\sim 100$ attoseconds $x$-ray spike. The peak power of the attosecond spike at the FEL saturation is about $2.3 \mathrm{GW}$, a factor of $\sim 20$ larger than the nearest side peaks and a factor of $\sim 200$ larger than the radiation power generated from an unmodulated electron beam.

\section{ACKNOWLEDGMENTS}

We would like to thank A. Zholents and W. White for useful discussions. This work was partially supported by the U.S. DOE Contract No. DE-AC02-76SF00515.

[1] E. L. Saldin, E. A. Schneidmiller, and M. V. Yurkov, Opt. Commun. 212, 377 (2002).

[2] A. A. Zholents and W. M. Fawley, Phys. Rev. Lett. 92, 224801 (2004).

[3] E. L. Saldin, E. A. Schneidmiller, and M. V. Yurkov, Opt. Commun. 237, 153 (2004).

[4] E. L. Saldin, E. A. Schneidmiller, and M. V. Yurkov, Opt. Commun. 239, 161 (2004).

[5] P. Emma, Z. Huang, and M. Borland, in Proceedings of FEL2004, Trieste, Italy, 2004 (Comitato Conferenze Elettra, Trieste, 2005), p. 333.

[6] A. A. Zholents and G. Penn, Phys. Rev. ST Accel. Beams 8, 050704 (2005).

[7] E. L. Saldin, E. A. Schneidmiller, and M. V. Yurkov, Phys. Rev. ST Accel. Beams 9, 050702 (2006).

[8] A. A. Zholents and M.S. Zolotorev, New J. Phys. 10, 025005 (2008).

[9] D. Xiang et al., Phys. Rev. ST Accel. Beams 12, 060701 (2009).

[10] A. A. Zholents, Phys. Rev. ST Accel. Beams 8, 040701 (2005).

[11] G. Geloni et al., Nucl. Instrum. Methods Phys. Res., Sect. A 583, 228 (2007).

[12] H. Merdji et al., Opt. Lett. 32, 3134 (2007).

[13] LCLS Conceptual Design Report No. SLAC-R-593, 2002.

[14] TESLA Technical Design Report No. TESLA FEL 200209, 2002.

[15] SPring-8 Compact SASE Source Conceptual Design Report, http://www-xfel.spring8.or.jp, 2005.

[16] C. Vozzi et al., Opt. Express 14, 10109 (2006); Opt. Lett. 32, 2957 (2007).

[17] A. W. Chao, Physics of Collective Beam Instabilities in High Energy Accelerators (Wiley, New York, 1993).

[18] S. Reiche, Nucl. Instrum. Methods Phys. Res., Sect. A 429, 243 (1999).

[19] W. M. Fawley, Nucl. Instrum. Methods Phys. Res., Sect. A 593, 111 (2008). 